\title{
El Diseño adaptado al entorno de la Industria Cultural y Creativa
}

\author{
Santamaría, Jorge Luis ${ }^{a}$ \& Lecuona-López, Manuel Ramón ${ }^{b}$ \\ ${ }^{a} \mathrm{PhD}$ candidate - Universitat Politècnica de València, Spain. george_lsa@hotmail.com, \\ ${ }^{b} \mathrm{PhD}$, Full Professor - Universitat Politècnica de València, Spain. mlecuona@dib.upv.es.
}

\begin{abstract}
Resumen
Los modelos de desarrollo tradicionales distan de la realidad de los tiempos modernos y se limitan ante nuevas posibilidades de cambio; por lo cual, las tecnologías y sus usos deben flexibilizar los procesos dando paso a un entorno proactivo que va de la mano de los integrantes del sector industrial y sus características. El desarrollo de las Industrias Culturales y Creativas [ICC] debe surgir en entornos fértiles donde las politicas, los profesionales especializados, el acceso a la tecnología, la innovación, las líneas de financiamiento y el mercado sean adecuados.
\end{abstract}

Las empresas conscientes de su entorno y de su evolución son capaces de adaptarse a nuevos escenarios, formas de producción y nuevas tecnologías para diversificar sus líneas de productos y hacer un control más eficiente en los procesos, desperdicio de recursos y la sustentabilidad. Por tanto, es necesario definir las caracteristicas de una visión de mercado e industria que se proyecte a futuro, y donde el diseño desarrolle, coordine y gestione los proyectos de diseño aplicados a emprendimientos de carácter cultural y creativo.

El paso natural de la industria a modelos flexibles donde la interconexión a través de redes, la comunicación entre personas y los objetos que nos rodean a través de la nube, genera muchas posibilidades en cuanto a productividad e innovación que mejoren la calidad de los productos o servicios y reduzcan el gasto en recursos y tiempo; por otro lado, el acercamiento del consumidor al proceso creativo viabiliza un enfoque personalizado de soluciones a problemas puntuales y una mayor satisfacción. Las empresas de menor tamaño pueden beneficiarse con los nuevos modelos de negocio, donde la interacción con otros sectores manufactureros o de servicios puede generar una gran red de trabajo adaptado a segmentos y necesidades especificas, para luego reconfigurase y direccionar su producción a otros segmentos y públicos si asi lo amerita el cambio constante del mercado.

Palabras clave: diseño, empresa, Industrias culturales y creativas, tecnología, transformación. 


\begin{abstract}
Traditional development models are far from the reality of modern times and are limited to new possibilities for change; therefore, technologies and their uses should be more flexible processes leading to a proactive environment that goes hand in hand with members of the industry and their characteristics. The development of cultural and creative industries [ICC] should arise in fertile environments where policies, specialized professionals, access to technology, innovation, financing lines and the market are adequate.

Conscious companies in their environment and their evolution are able to adapt to new scenarios, forms of production and new technologies to diversify their product lines and make more efficient process control, waste of resources and sustainability. It is therefore necessary to define the characteristics of a market vision and industry that is projected in the future, and where the design develop, coordinate and manage design projects applied to projects of cultural and creative character.

The natural step in the industry to flexible models where interconnection across networks, communication between people and objects around us through the cloud, creates many possibilities for productivity and innovation to improve the quality of products or services and reduce spending resources and time; on the other hand, the consumer approach to the creative process makes possible a personalized approach to solutions to specific problems and greater satisfaction. The smaller companies can benefit from new business models where interaction with other manufacturing or service sectors can generate a large network of work adapted to segments and specific needs, then reconfigured and direct their production to other segments and public if warranted the changing market.
\end{abstract}

Keywords: design, business, Cultural and creative industries, technology, processing.

\title{
1. Introducción
}

Las ICC como emprendimientos deben surgir de la mano de múltiples sectores, cuya estructura debe ser acorde a las posibilidades actuales y oportunidades futuras en un entorno cambiante. El diseño debe integrarse en cada empresa tomando en cuenta las características que configuran a cada sector de las ICC $^{12}$, pero también debe prever la evolución de esos entornos en aspectos como la tecnología, la comunicación, los procesos, y estructuras organizacionales. El camino a seguir debe ser labrado con las bases necesarias para su crecimiento; la innovación y calidad como base para la competitividad son herramientas que permiten la entrada a los mercados globales; por su parte, el diseño es partícipe de esta industria, aporta desde la creatividad y la gestión de proyectos de diseño al desarrollo de los sectores que componen las ICC.

\footnotetext{
${ }^{12}$ Los sectores de las ICC son: patrimonio cultural y natural; artes visuales y artesanía; presentaciones artísticas y celebraciones; libros y prensa; medios audiovisuales e interactivos; y, diseños y servicios creativos. Unesco $(2011$, p.19)
} 


\section{Entorno donde se desenvuelven las ICC}

Son diversos los temas que aportan en la conformación de un entorno favorable para el desarrollo de las ICC; la relevancia de cada tema dependerá del aporte que éste brinde al desarrollo de emprendimientos culturales y creativos; en este sentido, cada Estado y su gobierno debe proponer políticas que marquen pautas y tracen el camino para el desarrollo productivo, el apoyo a la cultura y a la creatividad. Solo con un marco de acción claro y viable, el diseño puede actuar y poner en marcha acciones y proyectos en beneficio de una industria en crecimiento.

Según UNESCO (2011, p.14) son seis las acciones de apoyo a las ICC:

\subsection{Competitividad e Innovación}

La mejora en las líneas de productos, sus procesos de producción y la necesidad de las empresas de ser flexibles y adaptarse a las exigencias actuales, son factores que las obligan a ser más competitivas. Los procesos, materias primas, la imagen, la función del producto, el enfoque hacia el público y mercado, etc., son factores a considerar al momento de lograr la competitividad; y esto se puede alcanzar desde una nueva visión de la empresa apoyada en la innovación creativa. en este sentido la UNESCO (2011) indica que el valor atribuido al producto o servicio para su comercialización ha pasado a ser un factor emocional, con importancia en cómo se lo comunica y lo que expresa en un proceso de vínculo con el consumidor (p.104). En este entorno innovador y creativo toma mayor fuerza el consumidor como parte activa del proceso; por lo que, los esfuerzos en innovación de los productos y su comercialización deben sustentarse en estudios de necesidades, gustos, tendencias e incluso se puede llegar a procesos cocreativos de diseño que integren diferentes fuentes de aporte como especialistas en otras áreas de conocimiento y los mismos usuarios.

\subsection{Recursos Humanos y Formación}

Las ICC al estar compuestas de múltiples sectores con diverso enfoque y origen, necesitan cubrir las distintas actividades derivadas de esta industria apoyándose en profesionales especializados que brinden el soporte e ideas que pongan en valor la creatividad y la cultura. UNESCO (2011) prioriza la necesidad de formar a emprendedores, creativos, gestores públicos y privados; y para esto, se debe tener claro el panorama de oportunidades, potenciales y capacidades que pueden desarrollarse; así también, las necesidades del contexto cambiante deben enfocado principalmente en las especificidades de los actores de esta industria y otros factores como lo social, el estado, estrategias y nuevas tecnologías (p.76). Ante este cúmulo de profesionales relacionados a las ICC, las universidades y centros de capacitación deben buscar construir el mejor perfil para formar individuos capaces de afrontar retos modernos, crear oportunidades y tener una visión global de futuro.

\subsection{Infraestructuras e Inversiones}

El medio donde se desarrollen las ICC debe ser adecuado para dicha actividad, ya sea enfocada en la creación, producción, promoción, comercialización; o cualquiera de las actividades propias de la industria, de las actividades complementarias y actividades relacionadas; que deben ser parte de una red de interacción y apoyo que permita su crecimiento. Para la UNESCO (2011) se requiere de instalaciones y equipos, talleres, espacios públicos, espacios de comercio y una logística e infraestructura administrativa que lo organice; es necesario un orden y planificación para lograr sinergias entre sectores productivos o clústeres y ser accesibles para todos los interesados (p.96). De esta necesidad han aparecido 
distintas iniciativas como las denominadas ciudades creativas ${ }^{13}$, estos modelos de desarrollo urbano basado en las ICC se han convertido en centros de cultura y creatividad donde se desarrollan actividades conjuntas con otros sectores como el turismo o la gastronomía, esto permite acercar tanto a ciudadanos como a foráneos para lograr interés y apoyo hacia el sector cultural y creativo.

\subsection{Financiación}

El apoyo económico es vital para esta industria, al conformarse de sectores emergentes o históricamente poco apoyados, es fundamental realizar un cambio en la perspectiva de apoyo a los emprendimientos asociados a las ICC; para lo cual, se debe fomentar políticas que se encaminen a generar líneas de crédito flexibles y ajustadas a las características de esta industria. Esto lo evidencia la UNESCO (2011) al manifestar que las ICC carecen de acceso a crédito debido a su alto nivel de riesgo, ya que su principal capital, la creatividad es considerada un intangible; esto limita el crecimiento de empresas, el desarrollo de proyectos y la creación de suficientes propuestas que sean atractivas hacia los mercados (p.86). El estado es el llamado a controlar esta situación con estrategias que promuevan el incentivo y apoyo por parte del sector financiero, en especial a las medianas, pequeñas y micro empresas.

\subsection{Marco Normativo}

El ámbito normativo debe ser entendido a nivel local con el fin de establecer oportunidades e igualdad para todos; pero también se debe proyectar hacia afuera, y es en ese entorno global donde debe existir equilibrio con las normativas internacionales fomentadas por organismos a nivel global o regional y que tienen la adhesión de varios países. Al respecto la UNESCO (2011) plantea que la necesidad de un marco normativo adecuado y obligatorio para todos los actores involucrados es una condición para el desarrollo de políticas de fomento que sean eficaces; además se necesita una integración con otras normativas relativas a la producción, los mercados y la distribución que influyen también en el desarrollo de las ICC, debiendo adaptarse a esta industria donde su materia prima, la cultura y la creatividad son intangibles (p.68). Contar con reglas claras para las ICC constituye un respaldo al asegurar las condiciones que les permitan desarrollarse y lograr los objetivos trazados.

\subsection{Desarrollo de Mercados}

El objetivo de las ICC para posicionar sus productos y servicios es llegar a los públicos de los mercados internos y externos. UNESCO (2011) afirma que el éxito de la oferta de las ICC depende de su aceptación por parte de los consumidores, ya que su incipiente paso por el mercado sería un fracaso económico; pero la clave se presenta en el momento de generar una sociedad que valore lo propio con el fin de fomentar una identidad que debe ser asimilada desde adentro para que sea creíble externamente (p.112). El trabajo es conjunto; la sociedad, los creativos y el estado deben proyectar una idea o concepto de identidad creíble en torno a la amalgama de sectores que conformen la ICC; que sea apreciado y consumido internamente, para llegar posteriormente a potenciales mercados internacionales.

El entorno macro donde se desarrolle esta industria debe ser el resultado de una apuesta elocuente con la realidad del país, región o ciudad donde se desarrolle, no se la puede entender como una estructura que se puede copiar de otras experiencias; será necesario un mapeo de los emprendimientos culturales y creativos que permita entender que sectores existen, cuales son los potenciales sectores a futuro, cuales son las fortalezas y debilidades, así como las particularidades de los sectores y actores que formaran esa industria.

\section{El entorno empresarial}

${ }_{13}$ Según Unctad (2010, p.40) Las ciudades creativas son "un conjunto urbano donde las actividades culturales de diferentes tipos son un componente de la economía de la ciudad y funcionamiento social" 
Al diseño como función de la empresa se lo debe enfocar desde su posición estratégica para el desarrollo de proyectos e innovación; para esto, se debe entender primero como es que la empresa debe manejarse con su entorno. Tasma y Loeb (1998) hablan de un cambio surgido en 1997 que nos traslada de una civilización tecnológica e industrializada, una civilización de flujos y la inmediatez, donde el individuo tiene el poder del cambio; y, el panorama de la empresa se configura bajo un fuerte dominio capitalista junto a factores de carácter: religioso, moral, estético, científico y tecnológico (p.p.136, 74). Esta situación plantea un cambio constante, un avance rápido y mediatizado donde la empresa debe interactuar con su entorno al ritmo que marca el mercado y la sociedad.

Finizio (2002) habla de un entorno basado en el mercado, donde los cambios producidos por la oferta y demanda se generan mediante una concepción clara de la realidad y una proyección en base a predicciones; esta visión proviene de la comprensión y estudio del mercado, el consumidor, la tecnología, el medio ambiente, entre otros; estos elementos en conjunto son los que dan pautas para lograr la evolución y el desarrollo de la empresa. Se debe tener una predisposición hacia el cambio y la flexibilidad; una empresa que quiera lograr comprender su entorno y adelantarse al futuro debe ser consiente que debe fortalecer su estructura, las herramientas operativas y su potencial innovador en procesos y productos (p.19).

Una empresa consiente de sus necesidades debe buscar la forma de comprender su entorno. Las pequeñas, medianas y microempresas [MIPYME] pueden ser las más afectadas al momento de identificar y estudiar estos elementos debido a sus limitados recursos; por lo que, es necesario entender esa flexibilidad y cambio como una nueva filosofía institucional que le lleve a la búsqueda de nuevas formas de trabajo en conjunto y apoyo con otros sectores y profesionales externos a la empresa; con respecto a empresas relacionadas con áreas culturales, Finizio (2002) indica que pueden estar conformadas por muchas variables y a su vez, pueden estar en conflicto entre sí; por tanto, los escenarios no son iguales para todas las empresas, siendo necesario que cada una se enfoque en buscar las estrategias acordes a su realidad (p.19)

Detectar los nuevos escenarios que la empresa debe enfrentar requiere de una capacidad de predecir los indicios que permiten entender hacia donde se mueve el mundo; la relación con factores culturales, políticos, sociales y de desarrollo del conocimiento son parte de estos indicios que generarán situaciones emergentes con las cuales poder trabajar. Para Finizio (2002, p.30), en estos escenarios "las empresas se mueven, o deben moverse, a través de un enfoque personalizado entre la globalización y la segmentación", siendo necesario una conjugación del diseño con todos las unidades de negocio de la empresa que cumplen un papel en la configuración del producto y que a su vez deben centrarse en identificar las necesidades y deseos del usuario; solo así se pueden llegar a soluciones específicas bajo una misma línea de trabajo conjunta.

Con un escenario definido por la empresa, el diseño debe enfocarse en el desarrollo de propuestas; y según lo planteado por Finizio (2002, p.30), son tres los ejes de impacto para el o los escenarios:

- Aplicación / función

- Tecnología / producto

- Consumidor / mercado

En cada escenario los ejes pueden variar o evolucionar; es decir, se puede optimizar el uso o función del objeto diseñado, así como, añadir nuevas características que lo mejoren; la tecnología avanza a pasos gigantes, modificando los entornos digitales y la industria en ciclos continuos cortos o medios. Así 
también, el consumidor evoluciona y se adapta a los cambios constantes, las necesidades y gustos se modifican al tiempo que se aprenden nuevas formas de uso y convivencia con los objetos y entornos.

Los ejes propuestos denotan una amplia visión global, donde se hace necesaria la presencia de especialistas en diversas áreas de conocimiento que apoyen el trabajo conjunto de la empresa. El acceso a los especialistas o fuentes de información especializada puede ser visto como un gasto importante por una empresa, por lo que se debe buscar facilidades que permitan un beneficio común a todos los sectores que conformen las ICC; en este punto se vuelve aún más significante que los sectores culturales y creativos formen sinergias que los fortalezcan al momento de interactuar con otras áreas o sectores de apoyo.

Por otro lado, Tasma y Loeb (1998, p.p.136,74) describen este nuevo escenario en base a puntos de giro como:

- Constantes cambios cíclicos y mutaciones

- Una nueva distribución del poder y la aparición de nuevas tecnologías de red

- La ciencia superando a la ficción

- Las maquinas compitiendo y superando las capacidades humanas

- Maximizar los resultados empresariales en base a mantener la rentabilidad de sus dividendos

- El poder de los que controlan el flujo de dinero, los servicios, la información, los bienes, etc.

- Perdida de la propiedad intelectual de las creaciones debido a la reproducción no controlada de ideas.

- Nuevos caminos y expresiones artísticas.

La vertiginosa dinámica presente en el escenario en que se mueve la empresa, hace que deban buscar nuevas opciones y oportunidades que les permita integrarse a los estados actuales de la sociedad y el mercado; por tanto, son las nuevas formas de producción y asociación productiva las que deben evolucionar y ser adaptadas por las empresas.

Esta estructura actual de los mercados y la sociedad presenta un cambio concordante hacia la masificación, el acceso a la información y el conocimiento gracias a la internet y las formas de comunicación de la era digital; los cambios a partir de 1997 (Tasma y Loeb, 1998), representan una búsqueda de armonía para todos, el nacimiento de las redes de información, la gestión del tiempo y la creación de tribus o simbiontes en la sociedad (p.134). El modelo en que actualmente se desarrolla la sociedad describe una división entre ricos y pobres denominada cultura tribal (Fig. 1.); esta estructura marca una diferencia entre los que tienen y los que no, y determina la pauta para la segmentación de los mercados con los que se mueven los mercados actualmente, la especialización según gustos, preferencias y necesidades lleva a las empresas a generar productos y servicios cada vez más especializados y específicos para los públicos. 


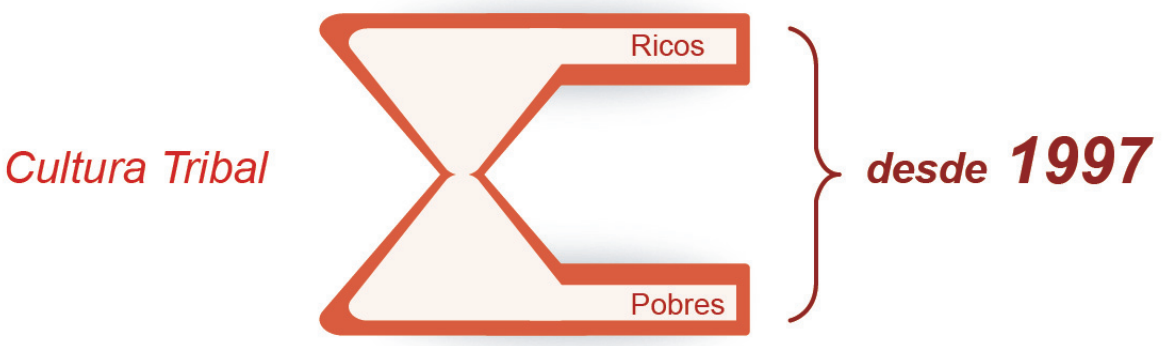

Fig. 1 Distribución de la sociedad desde 1997 (Cultura tribal). Tasma y Loeb (1998)

Esta visión propia de los modelos de negocio tradicionales como la gran industria o las MIPYME, pone en valor la necesidad de adaptarse a un estrato $u$ otro al momento de generar propuestas de nuevos productos y servicios según el enfoque de la empresa; el diseño desde esta perspectiva, debe centrarse en buscar soluciones funcionales para cada nivel, generando una diversidad de opciones adaptadas a cada situación y posibilidades de los consumidores. Además de los segmentos en cuanto a nivel socioeconómico, también se presenta el entorno socio-cultural; Finizio (2002) destaca la importancia de marcar el mercado objetivo en base a las señales que se detectan en este entorno para poder definir el alcance del mercado, sea este local, regional o global; y determinar características y requerimientos que serán aplicados al diseño (p36). Cada uno de los segmentos o mercados determinan características específicas del producto, sin afectar otros factores comunes a todo proyecto de diseño: social, responsable con el medio ambiente, de calidad, ergonómico, funcional, intuitivo, etc.

\section{Nuevas configuraciones de empresa}

La industria a través de los años ha evolucionado sus formas de producción debido a los cambios sociales, culturales, avances en el conocimiento y las nuevas maneras de consumir; estos cambios del entorno externo también afectan al entorno interno de la empresa y su estructura. La empresa como una organización se estructura por unidades que trabajan en conjunto para lograr objetivos; Finizio (2002) destaca el concepto de red de empresa como alternativa a las estructuras tradicionales jerárquicas donde todas las funciones se ejecutan dentro de esta; el sistema de redes permite desarrollar funciones externamente pero con una guía y control desde la empresa, siendo esto un sistema flexible con resultados competitivos y eficaces (p.36). Las empresas deben buscar opciones para ser competitivas; limitantes de tamaño, geográficas, tecnológicas, políticas, etc., son factores que deben solventarse gracias a acciones estratégicas acordes a la situación de la empresa.

La visión actual de empresas nacidas de startups o emprendedores que se abren paso de forma independiente crea un panorama competitivo desde el momento mismo que nacen las ideas y que buscan salir adelante; inversores o alianzas son necesarias desde estados incipientes del negocio para su éxito, y debe ser una constante en el futuro crecimiento de la misma.

En la actualidad; la llegada de la industria 4.0 marca un nuevo hito en la visión de la industria, basada en el Internet de las cosas (IoT) por sus siglas en inglés, permiten una conectividad inteligente y la economía de recursos; siendo los factores la clave de esta industria (BMBF, 2014, p.16):

- Producción ampliamente personalizada dentro de entornos productivos de gran flexibilidad. 
- Integración de clientes y socios en etapas tempranas de diseño y generación de valor.

- Vinculación entre la producción y servicios de alta calidad para generar "productos híbridos"

Este nuevo panorama permite el desarrollo de nuevos productos, servicios, procesos, comunicación y emprendimientos que requieren de profesionales especializados en diferentes áreas del diseño, además de servicios derivados de esta actividad creativa.

La industria 4.0 se integra acorde a los cambios sociales y culturales de pensamiento sobre nuestro entorno y el lugar que ocupamos en este mundo, la preocupación por los recursos y estar cada vez mejor comunicados son valores importantes de este modelo productivo; la convergencia del mundo real y el digital permite optimizar las experiencias y ampliarlas. Una definición derivada de la industria 4.0 es la referente a las industrias inteligentes:

Son industrias que tienen un alto grado de flexibilidad en la producción, en términos de las necesidades del producto (especificaciones, calidad, diseño), volumen (lo que se necesita), el momento (cuando sea necesario), eficiencia de los recursos y los costes (lo que se requiera), pudiendo sintonizar (de buena forma) las necesidades del cliente y hacer uso de toda la cadena de suministro para la creación de valor. Se activa por un enfoque de red-centralizada, haciendo uso del valor de la información, impulsada por las TIC y las últimas técnicas de fabricación disponibles. (Smart Industry Report, 2014)

Esta definición permite tener una idea clara de las posibilidades que se derivan del uso de la tecnología de punta y la productividad, sobre todo al momento de ser versátiles en un entorno de ciclos cortos y cambiantes.

\section{El diseño como dinamizador de las ICC en un entorno de cambios constantes}

De este entorno y configuración actual de la industria, se puede entender la necesidad de ver al diseño de una manera diferente a lo ya visto, los modelos tradicionales se vuelven obsoletos debido a su rigidez estructural que limita su migración a diferentes líneas de producción; otro factor es la cantidad de inversión que representaría en los modelos tradicionales implementar nueva infraestructura, que conjugada con la existente obliga a un crecimiento en cuanto a espacio y capital de producción que muchas MIPYMES no se lo pueden permitir.

Esto pone aún más en evidencia la necesidad de generar lazos entre distintas actividades productivas que se complementen y generen una simbiosis de proyectos con mutuos beneficios a las MIPYME o grandes empresas que intervengan. Una estructura funcional y adaptada para la tecnología, el cambio constante, el trabajo interactivo, la interconexión de redes y los actores o consumidores presentes, conforman los ejes que definirían una empresa de la industria 4.0; además, esto permitiría fomentar la producción local mediante unidades productivas que puedan solventar necesidades específicas sin tener que recurrir a producción en masa con su consecuente desperdicio y poca conciencia en el ámbito de la producción sustentable. El diseño por su parte, se integra en el proceso de la empresa desde la ideación de proyectos hasta el momento mismo de la experiencia entre usuario y producto; ya que en sí, el diseño configura esa experiencia y debe identificar las respuestas obtenidas.

Manzini (2015) plantea cómo se debe poner en práctica la capacidad de diseñar en función a dos ejes: el eje de actores y competencias; y el eje de motivaciones y expectativas, de donde surgen cuatro actividades:

- Organizaciones base, personas no expertas que impulsan proyectos de diseño desde la detección de necesidades o problemas 
- Activistas culturales, personas interesadas en actividades, su desarrollo y promoción con un interés cultural

- Diseño y agencias de comunicación, expertos encargados en el desarrollo de productos, servicios y sistemas de comunicación

- Diseño y agencia tecnológica, expertos multidisciplinarios con un perfil técnico para la solución de diversos problemas; abierto a nuevas experiencias y procesos de diseño. (p.p.50-55)

Esta perspectiva ubica al diseño en diferentes niveles de interacción dentro de la sociedad, enfocado siempre en la propuesta de acciones; desde las organizaciones base y activistas culturales se puede obtener la información necesaria para el desarrollo de proyectos, retroalimentación de información o experiencias y la validación de los diseños; mientras que las agencias tecnológicas y de comunicación son las encargadas de poner en marcha las acciones y propuestas de diseño. Los factores existentes permiten identificar posibles configuraciones para el papel que el diseño debe cumplir en este tipo de empresas, siendo la propuesta del enfoque hacia las ICC el siguiente:

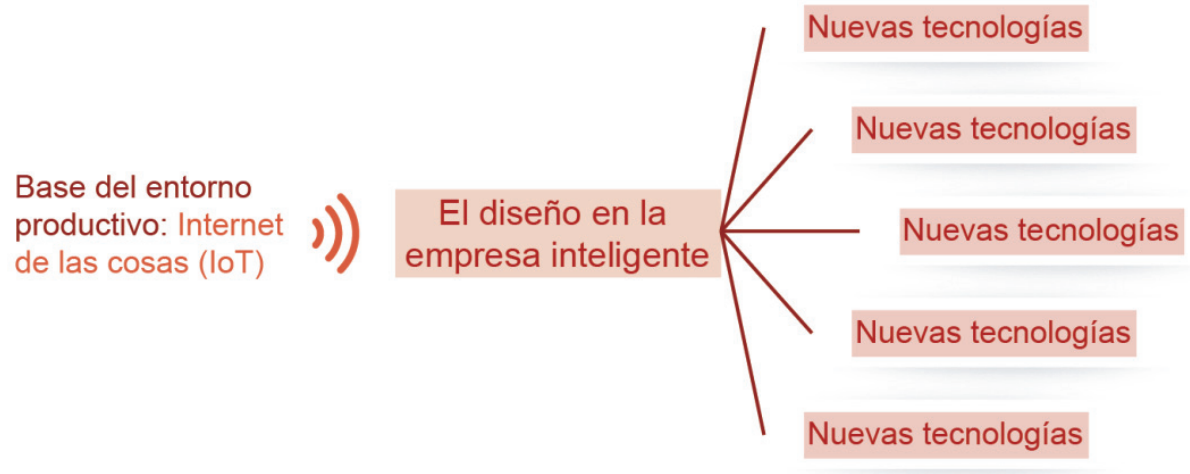

Fig. 2 Entorno productivo para las ICC.. Elaboración propia.

De este entorno se puede plantear acciones desde el diseño cómo:

- Integración de apps especializadas y dispositivos móviles para el desarrollo de ideas, bocetos, prototipos y el trabajo en equipo de la empresa.

- Trabajo en espacios amplios y configurables, que puedan ser desde un taller de experimentación hasta un salón de reuniones con el fin de generar acciones comunes de creación.

- Comunicación continua entre las unidades de negocio para la solución de problemas, control y gestión del diseño.

- Equipamiento para prototipado rápido y series cortas que permita flexibilizar la producción y abrir nuevas líneas de productos (dentro de la empresa o como servicio de diseño externo)

- El diseñador como gestor de experiencias aumentadas a los productos, mediante propuestas de servicios asociados (on-line) 
- El diseñador como promotor del acercamiento y fidelización creativa de los usuarios a través de servicios complementarios (DiY)

- Gestión centralizada de procesos mediante la interconexión de equipos y dispositivos en la empresa.

- Coordinación y supervisión de proyectos mediante trabajo conjunto con otras empresas (on-line)

- Seguimiento de resultados mediante la retro alimentación de experiencias de usuarios que se contrasten con experiencias logradas en los procesos de desarrollo.

- Diversificación estratificada según el segmento o mercado, pudiendo identificar necesidades específicas de sectores de menos recursos o integrando la personalización del diseño como valor agregado a sectores más pudientes.

\section{Conclusiones}

En un entorno cambiante y flexible donde es necesario la respuesta y reacción inmediata, los modelos tradicionales jerárquicos presentan dificultades en la integración del diseño como eje de construcción de propuestas innovadoras, las acciones del diseño en la empresa deben tener una interacción constante con el resto de departamentos relacionados a la producción y generación de proyectos para lograr respuestas inmediatas y eficientes; esto hace necesario evitar perder el tiempo en una comunicación vertical sujeta a la toma de decisiones de los altos mandos, sino una comunicación abierta y trabajo en equipo.

El diseño debe tener su lugar en la empresa, sea como parte de esta o de forma externa; y su trabajo debe abarcar los procesos y actividades de la empresa valiéndose de todos los recursos posibles para dar apoyo y guiar los proyectos hasta su fin. Las múltiples actividades que el diseño debe cumplir en el actual panorama productivo, obliga a las medianas, pequeñas y microempresas a considerar una forma de integrarlo, siendo una posición viable el trabajo en red como una unidad externa de la empresa que gestiones sus procesos creativos y productivos, esto generaría mayor competitividad y posibilidades de ampliar su producción; aún más si desde el diseño se proponen servicios complementarios para la empresa y se refuerza su capacidad productiva.

\section{Referencias}

CONFERENCIA DE LAS NACIONES UNIDAS SOBRE COMERCIO Y DESARROLLO [UNCTAD]. (2010). Economía Creativa: Una opción factible de desarrollo. $<$ http://unctad.org/es/Docs/ditctab20103_sp.pdf $>$ [Consulta: 15 de febrero de 2015]

FEDERAL MINISTRY OF EDUCATION AND RESEARCH (BMBF) DIVISION INNOVATION POLICY ISSUES. (2014.) The new High-Tech Strategy Innovations for Germany. Berlín. $<$ https://www.bmbf.de/pub/HTS_Broschuere_engl_bf.pdf> [Consulta: 10 de marzo de 2015]

FINIZIO, G. (2002). Design \& Management. Milano: Skira.

MANZINI, E. (2015). Cuando todos diseñan: Una introducción al diseño para la innovación social. Madrid: Gráficas Muriel

ORGANIZACION DE LAS NACIONES UNIDAS PARA LA EDUCACION, LA CIENCIA Y LA CULTURA [UNESCO]. (2011). Politicas para la creatividad. Guía para el desarrollo de las industrias culturales y creativas. Argentina.

$<$ http://www.unesco.org/new/fileadmin/MULTIMEDIA/HQ/CLT/images/UNESCOCulturalandCreativeIndustriesgui de_01.pdf $>$ [Consulta: 12 de marzo de 2015] 
SMART INDUSTRY INITIATIVE. (2014). SMART INDUSTRY DUTCH INDUSTRY FIT FOR THE FUTURE. Holanda. <http://www.smartindustry.nl/wp-content/uploads/2014/07/Opmaak-Smart-Industry.pdf> [Consulta: $10 \mathrm{de}$ marzo de 2015]

TASMA, S. Y LOEB, F. (1998). Et si on Remettait les computeurs à zéro? Hypothèses pour des futurs probables. Paris: VIA 